\title{
Application of Wavelet Denoising Method in Temperature Data Processing of Coal and Gas Outburst Simulation Test
}

\author{
Cao $\mathrm{Jie}^{1,2, \mathrm{a}^{*}}$ \\ ${ }^{1}$ Gas Research Branch, China Coal Technology Engineering Group Chongqing Research Institute, \\ Chongqing 400037, China \\ ${ }^{2}$ State Key Laboratory of the Gas Disaster Detecting Preventing and Emergency Controlling, \\ Chongqing 400037, China \\ acqcaoj@126.com
}

\begin{abstract}
The analysis of the evolution of various parameters in the roadway during the process of outburst through the physical simulation test is the development trend of studying the mechanism of the disaster of coal and gas outburst. Because the temperature data obtained by the test carries a lot of noise, this paper chose the threshold denoising method, and used Matlab to analyze the effects of different wavelet decomposition levels and threshold selection methods on the denoising effect. Finally, the wavelet denoising of temperature data was used to obtain more realistic experimental data. By analyzing the temperature variation at different locations, it can be found that the closer the location of the main roadway to the outburst port, the more rapid the temperature change. Due to the attenuation of shock wave energy, the relative change peak value in the temperature of the branch roadway is only about $1 / 4$ that of the main roadway.
\end{abstract}

Keywords: Coal and gas outburst, Simulation test, Temperature data, Wavelet denoising

\section{Introduction}

Coal and gas outburst accidents are one of the most serious disasters that have disturbed the safe production of underground coal mines. Once an outburst accident occurs, the high-pressure gas in the coal seam and the broken coal (rock) can migrate into the roadway space from tens to hundreds meters, resulting in damage to the underground facilities and triggering gas explosions, resulting in a huge dynamic effect. By conducting physical simulation tests, the changes in parameters such as gas pressure, temperature and gas concentration in the roadway during the process of outburst and the distribution law of pulverized coal are studied, the mechanism of outburst impact-induced disasters are researched, which can provide guidance for outburst disaster prevention and control.

Due to the limitations of experimental conditions, previous researchers have conducted a lot of research on the changes in the internal data of the coal seam during the outburst experiment process [1 4], few studies on data changes in the roadway have conducted. These studies focus on gas pressure changes in the roadway. For example, Wang et al. $[5,6]$ used the experimental device to conduct an outburst disaster test, and analyzed the changes of the gas pressure under different roadway distribution conditions. Based on the theories of coal spherical shell failure and the pneumatic conveying, the coal and gas flow and the transportation mechanism are analyzed by Zhao et al. [7]. In this kind of research, there is almost no analysis of temperature data. And a large amount of noise is involved in the acquisition of data, which affects the acquisition accuracy and resolution of the sensor. In order to better study the trend of temperature changes in the roadway during the process of coal and gas outbursts, this paper proposes to use wavelet analysis to eliminate noise and restore signals to provide basis for further research.

\section{Type and characteristics of wavelet denoising}

The traditional denoising methods mainly include linear filtering and nonlinear filtering, but often result in distortion of the useful signal while filtering out noise. That increases the entropy of the signal and makes it impossible to characterize the nonstationarity of the signal. Denoising using wavelet 
transform has multi-resolution, and has local characteristics in both time-frequency domain, which is suitable for analyzing non-stationary signals. There are four commonly used wavelet denoising methods.

Wavelet decomposition and reconstruction method denoising. In 1988, Mallat [8] proposed the concept of multi-resolution analysis and presented a fast algorithm for wavelet decomposition and reconstruction. This method is mainly applicable to the case of deterministic noise when the frequency bands of useful signals and noise are separated from each other. The advantage of this method is that the algorithm is simple and clear, and the calculation speed is fast. However, when the frequency bands of useful signals and noise overlap each other (for example, the signal is mixed with white noise), the effect is not ideal.

Nonlinear wavelet transform threshold denoising. Donoho et al. $[9,10]$ proposed a wavelet threshold denoising method and studied the method of detecting real signals under white gaussian noise. This method is mainly applied to the situation where the signal is mixed with white noise. The advantage is that the noise is almost completely suppressed, and the characteristic peak point reflecting the original signal is well preserved, and is the most widely used one in many wavelet denoising methods. The disadvantage is that in some cases, such as at the discontinuous point of the signal, a pseudo-Gibbs phenomenon occurs after denoising.

Translation invariant wavelet denoising. This method is mainly applied to the situation where the signal is mixed with white noise and contains several discontinuities. It is an improvement based on the threshold method. Its advantages can effectively remove the pseudo-Gibbs phenomenon generated at the discontinuity point of the signal in the denoising of the threshold method, and show a better visual effect than the threshold method. The denoising method can obtain smaller root mean square error than the threshold method, and the signal-to-noise ratio is also improved. The disadvantage is that the calculation speed is relatively slow.

Modulus Maximum Method Denoising. This method is mainly applied to the situation where the signal is mixed with white noise and the signal contains more singular points. This method can effectively preserve the singular point information of the signal while denoising. The denoised signal has no unwanted oscillation. However, its calculation speed is very slow, and it is usually tens of times slower than the previous methods. At the same time, using this method to denoise, the choice of wavelet decomposition scale is very important. At small scale, the wavelet coefficients are greatly influenced by noise, resulting in many pseudo-extreme points; large scale will cause the signal to lose some important local singularities [11].

\section{Temperature Data Collecting of Coal and Gas Outburst Disaster Test}

The experiments were operated under the independent self-development coal and gas outburst dynamic simulation test device [12], as shown in Fig.1.

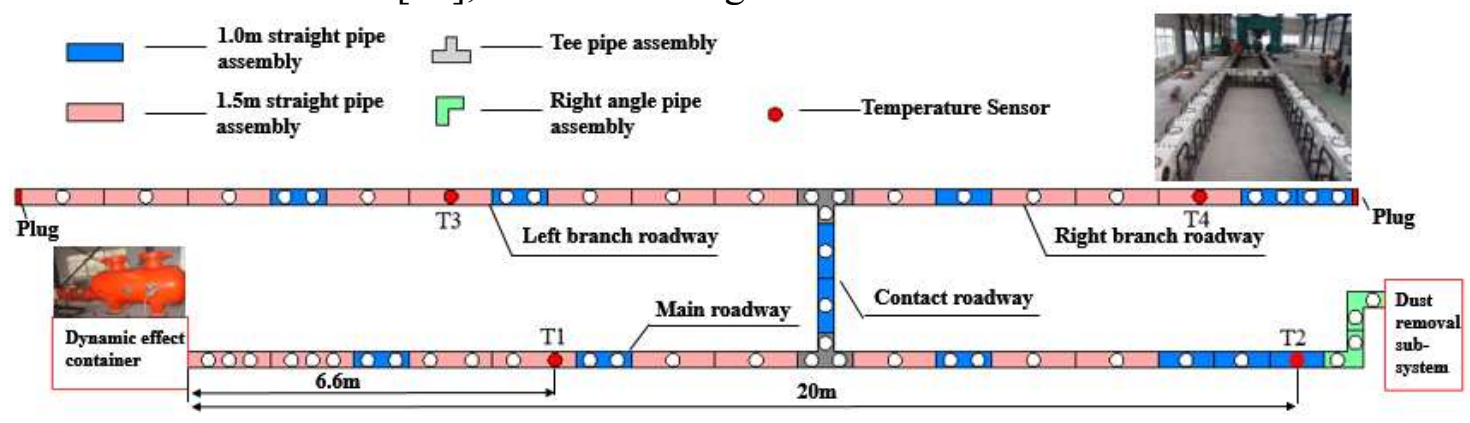

Fig. 1. Test and sensor layout schematic.

The coal samples of outburst coal seam M8 in Chongqing Yuyang Coal Mine in China were selected as experimental objects. In order to ensure the safety, high-purity $\mathrm{CO}_{2}$ gas was used instead of methane as the experimental gas. Under the gas pressure of $0.35 \mathrm{MPa}$, launched the rupture disks of device outburst port to simulate the coal and gas outburst, and the data monitoring system was started to collect the temperature signal in the roadway. The location of the temperature sensor is also shown in 
Fig. 1, the theoretical measurement range is $0-50^{\circ} \mathrm{C}$. Automatic collection of data improves efficiency and data richness, but these sampled signals are inevitably polluted by noise, as shown in Fig. 2 .

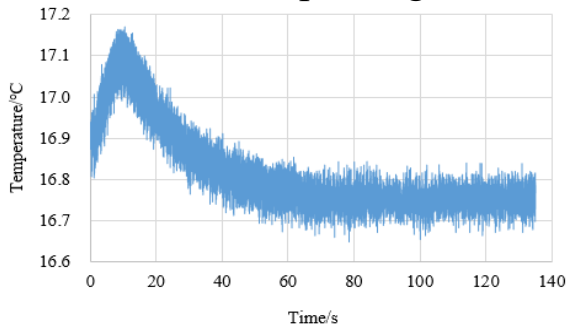

(a) $\mathrm{T} 1$

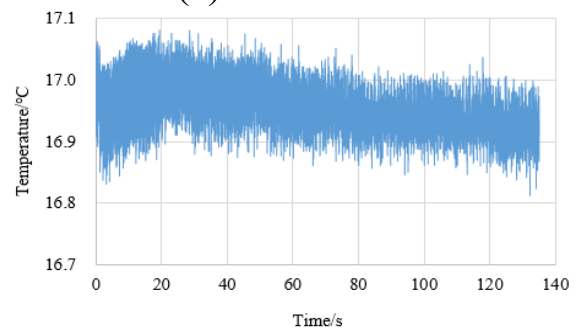

(c) $\mathrm{T} 3$

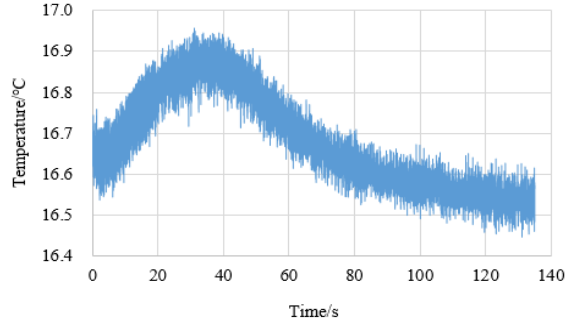

(b) $\mathrm{T} 2$

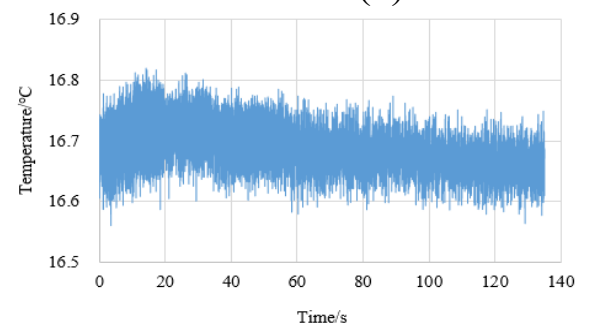

(d) $\mathrm{T} 4$

Fig. 2 The diagram of temperature sensor acquisition data

\section{Application of temperature data denoising}

According to the above summary of the wavelet denoising method and the characteristics of temperature data in the roadway during coal and gas outburst, the nonlinear wavelet transform threshold method is used to denoise the temperature data.

Principle of nonlinear wavelet transform threshold denoising. Assume that a one-dimensional signal model containing noise can be represented as

$$
s(n)=f(n)+\sigma^{*} e(n)
$$

Where, $s(n)$ is a signal containing noise; $f(n)$ is a useful signal; $e(n)$ is a noise, $n$ is an equally spaced time; and $\sigma$ is a noise level. The purpose of wavelet denoising is to suppress the portion of noise in the signal $s(n)$ and reproduce the signal $f(n)$. The threshold denoising method is divided into the following three steps:

(1) Calculate an orthonormal wavelet transform with noisy signals. Select the appropriate wavelet and wavelet decomposition layer $j$, and use the Eq.2 to carry out the wavelet decomposition of the value $\mathrm{j}$-layer to get the corresponding wavelet decomposition coefficients.

$$
\left\{\begin{array}{l}
c_{j, k}=\sum_{n} c_{j-1, n} h_{n-2 k} \\
d_{j, k}=\sum_{n} d_{j-1, n} g_{n-2 k}
\end{array}(k=0,1,2, \mathrm{~L}, N-1)\right.
$$

Where, $c_{j, k}$ is the scale factor; $d_{j, k}$ is the wavelet coefficient; $h, g$ is a stack of orthogonal image filterbanks; $j$ is the number of decomposition layers, and $N$ is the discrete number of points.

(2) Making threshold processing for the wavelet coefficients obtained by the decomposition, and there are two processing methods:

The hard threshold method, it means that when the absolute value of the wavelet coefficient is greater than the threshold, it remains unchanged; otherwise it is zero.

$$
s=\left\{\begin{array}{l}
x,|x|>T \\
0,|x| \leq T
\end{array}\right.
$$

The soft threshold method, when the absolute value of the wavelet coefficient is greater than the threshold, becomes the difference between the two; otherwise it is zero, and its expression is: 


$$
s=\left\{\begin{array}{l}
\operatorname{sign}(x)(|x|-T),|x|>T \\
0,|x| \leq T
\end{array}\right.
$$

(3) Wavelet inverse transform is performed, and the threshold-processed wavelet coefficients are reconstructed by the following equation, and the original signal estimated value is recovered.

$$
c_{j-1, n}=\sum_{n} c_{j, n} h_{k-2 n}+\sum_{n} d_{j, n} g_{k-2 n}
$$

Calculation cases. Using the one-dimensional wavelet analysis tool of Matlab software, the temperature data $\mathrm{T} 1$ obtained by the experiment is denoised. The widely used in engineering db1 wavelet is used to decompose 1, 2, 3, and 4 layers, and soft thresholds are used to use unbiased likelihood estimation. Four kinds of thresholds such as rigorous sure, heuristic sure, minimax, and fixed form threshold are used to denoise the signal. The effect of denoising the signal using the four thresholds is basically the same for the temperature data collected in the roadway. Fig. 3 shows the denoising results of different decomposition layers. It can be seen that the denoising effect becomes better with the increase of the wavelet decomposition level, but the improvement of the denoising effect after the decomposition level is more than 3 layers is not obvious. So the 3 layers decomposition is selected for temperature data processing is more reasonable.

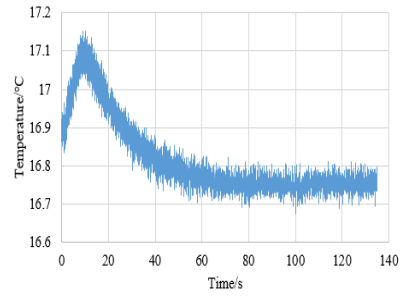

(a)1 layer

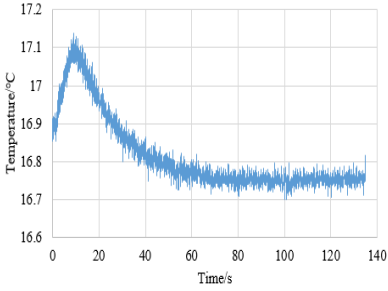

(b)2 layers

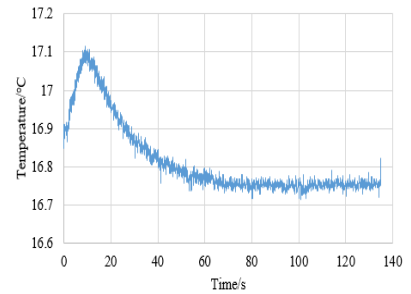

(c)3 layers



(d)4layers

Fig.3 The signal denoising in different decomposition layers

The wavelet denoising analysis is used to process the temperature data collected by the test to obtain the temperature variation at different positions of the roadway, as shown in Fig.4.
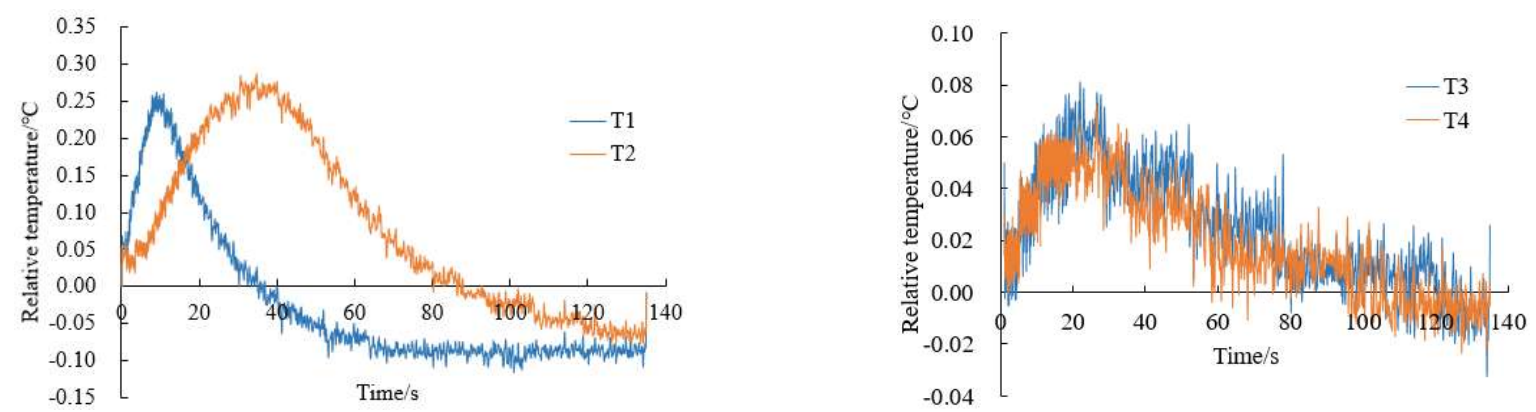

Fig.4 The relative temperature variation in different loaction in roadway

It can be seen that the closer the position of the outburst port in the main roadway, the more rapid the temperature change. At a distance of $6.6 \mathrm{~m}$ and $20 \mathrm{~m}$ from the outburst port, the phase with a relative temperature change of more than $0^{\circ} \mathrm{C}$ lasts for about $36.4 \mathrm{~s}$ and $86.3 \mathrm{~s}$, respectively. The left and right lane temperature sensors are arranged at the same distance from the outburst port, so the temperature variation and the law are basically the same. The peak value of the relative temperature variation in the main roadway is $0.25^{\circ} \mathrm{C}$, while the relative change in the temperature within the branch is due to the attenuation of the shock wave energy, and the relative variation peak value is only about $0.065^{\circ} \mathrm{C}$.

\section{Summary}


This paper analyzed the principle of wavelet denoising, chose the threshold denoising method, and used Matlab to analyze the effects of different wavelet decomposition levels and threshold selection methods on the denoising effect. And then the wavelet denoising of temperature data was used to obtain more realistic experimental data. By analyzing the temperature variation at different locations, it can be found that the closer the location of the main roadway to the outburst port, the more rapid the temperature change. Due to the attenuation of shock wave energy, the relative change peak value in the temperature of the branch roadway is only about $1 / 4$ that of the main roadway.

\section{Acknowledgments}

This study was financially supported by National Natural Science Foundation of China (51774319, 51574280), Chongqing science and technology innovation leader talent support program (CSTCKJCXLJRC14).

\section{References}

[1] J. Sobczyk, The influence of sorption processes on gas stresses leading to the coal and gas outburst in the laboratory conditions, Fuel. 90(2011)1018-1023.

[2] Q.Y. Tu, Y.P. Cheng, P.K. Guo, J.Y. Jiang, L. Wang, R. Zhang, Experimental study of coal and gas outbursts related to gas-enriched areas, Rock Mech. Rock Eng. 49(2016)3769-3781.

[3] K. Gao, Z.G. Liu, J. Liu, Design of outburst experiment device based on similar simulation and geomechanical model test and its application, Rock and Mechanics. 36(2015)711-718.

[4] J.P. Tang, Y.S. Pan, S.L. Yang. Experimental study of coal and gas outburst under tridimensional stresses, Chinese Journal of Rock Mechanics and Engineering. 32(2013)960-965.

[5] K. Wang, A.T. Zhou, J.F. Zhang, P. Zhang. Real-time numerical simulations and experimental research for the propagation characteristics of shock waves and gas flow during coal and gas outburst, Safety Sci. 50(2012)835-841.

[6] A.T. Zhou, K. Wang, L. Wang, F. Du, Z.L. Li, Numerical simulation for propagation characteristics of shock wave and gas flow induced by outburst intensity. Int. J. Min. Sci. Technol. 25(2015)107-112.

[7] W. Zhao, Y.P. Cheng, P.K. Guo, K. Jin, Q.Y. Tu, H.F. Wang, An analysis of the gas-solid plug flow formation new insights into the coal failure process during coal and gas outbursts Original, Powder Technol. 305(2017)39-47.

[8] S. Mallat, Theory for multi-resolution signal decomposition: The wavelet representation, IEEE Transactions on Pattern Analysis and Machine Intelligence. 11(1989)674-693.

[9] D.L. Donoho, De-nosing by soft-thresholding, IEEE Transactions on Information Theory. 41(1995)613-627.

[10] D.L. Donoho, I. Johnstone, Wavelet shrinkage asymptopia, Journal of Royal Statistical Society. 57(1995)301-369.

[11] Y.H. Peng, Wavelet transform and engineering application, Science Press, Beijing,1999, pp.59-62.

[12] J. Cao, H.T. Sun, L.C. Dai, D.L. Sun, B. Wang, F.T. Miao, Simulation research on dynamic effect of coal and gas outburst. Journal of China University of Mining \& Technology. 47(2018)100-107. 